\title{
A COMPATIBILITY THEOREM FOR TWO POINT BOUNDARY PROBLEMS
}

\section{E. J. PELLICCIARO}

Let $P$ be an $n$ by $n$ matrix whose elements are complex valued functions continuous in $(t, \lambda)$ for $t$ in the closed interval $[a, b]$ and $\lambda$ in the closed disk $R=\left\{\lambda:\left|\lambda-\lambda_{0}\right| \leqq r, r>0\right\}$ in the complex plane. Consider the $n$-vector differential equation

$$
d y / d t=P y
$$

together with the homogeneous $n$-vector two point boundary conditions

$$
U y \equiv A y(a)+B y(b)=0,
$$

where $A$ and $B$ are $n$ by $n$ matrices the elements of which are continuous complex valued functions on $R$.

The compatibility of (1), (2) at $\lambda \in R$ is defined to be the maximum number of linearly independent solutions of (1), (2) corresponding to $\lambda$. Let $y_{1}, \cdots, y_{n}$ be a fundamental set of solutions of (1) continuous in $(t, \lambda)$ for $t \in[a, b]$ and $\lambda \in R$, hence uniformly continuous there. A necessary and sufficient condition for the compatibility of (1), (2) at $\lambda$ to be $k$ is that the rank of the $n$ by $n$ matrix $V(\lambda)$ with elements $U_{i} y_{j}(\lambda)$ be of rank $n-k$. It is known [1], [2] that if the compatibility of (1), (2) is constant in some neighborhood of $\lambda_{0}$ and $x(t)$ is a solution of (1), (2) for $\lambda=\lambda_{0}$, then there exists a solution $x(t, \lambda)$ of (1), (2) which is uniformly continuous in $(t, \lambda)$ for $t \in[a, b]$ and $\lambda$ in some neighborhood of $\lambda_{0}$ and which is such that $x\left(t, \lambda_{0}\right)=x(t)$ so that $x(t, \lambda) \rightarrow x(t)$ uniformly on $[a, b]$. The question, a natural one, as to what can be said in case the compatibility is not constant in any neighborhood of $\lambda_{0}$ was brought to the attention of the author by W. M. Whyburn [3]. An answer to the question is in the following theorem.

THEOREM 1. If the compatibility of (1), (2) at $\lambda_{0}$ is $k_{0}>0$ and is not identically zero in any deleted neighborhood of $\lambda_{0}$, then there exists an integer $k$ with $0<k \leqq k_{0}$, an infinite sequence $\left\{\lambda_{m}\right\}$ of distinct values of $\lambda$ converging to $\lambda_{0}$ at each $\lambda_{m}$ of which the compatibility of (1), (2) is $k$, and correspondingly $k$ linearly independent sequences $\left\{x_{1}\left(t, \lambda_{m}\right)\right\}$, $\cdots,\left\{x_{k}\left(t, \lambda_{m}\right)\right\}$ of solutions of (1), (2) for $\lambda=\lambda_{m}$ which converge uni-

Received by the editors March 20, 1967. 
formly on $[a, b]$ to some $k$ linearly independent solutions $x_{1}(t), \ldots$, $x_{k}(t)$ of (1), (2) for $\lambda=\lambda_{0}$.

Proof. $V(\lambda)$ as defined above is continuous on $R$, implying the compatibility of (1), (2) is less than or equal to $k_{0}$ at each $\lambda$ in some neighborhood of $\lambda_{0}$. There exists an in teger $k, 0<k \leqq k_{0}$, and an infinite sequence $S$ of distinct values of $\lambda$ converging to $\lambda_{0}$ at each term of which the compatibility of (1), (2) is $k$. The case $k=n$ is disposed of as being trivial since in this case the rank of $V(\lambda)$ is zero for $\lambda=\lambda_{0}$ and every $\lambda \in S$, implying $y_{1}, \cdots, y_{n}$ are linearly independent solutions of (1), (2) for $\lambda=\lambda_{0}$ and every $\lambda \in S$. For the case $k<n$ then, since $V(\lambda)$ is $n$ by $n$ with $n$ finite, it is possible to select an infinite subsequence $\left\{\lambda_{m}\right\}$ of $S$ together with two finite subsequences $i_{1}, \cdots, i_{n-k}$ and $j_{1}, \cdots, j_{n-k}$ of the finite sequence $1, \cdots, n$ which for every $m$ satisfies (i) the $n-k$ by $n-k$ matrix $\delta_{m}=\left[U_{i_{\alpha}} y_{j_{\beta}}\left(\lambda_{m}\right)\right]$ is such that det $\delta_{m} \neq 0$ and (ii) if $h_{1}, \cdots, h_{n-k}$ is any subsequence of $1, \cdots, n$, then the $n-k$ by $n-k$ matrix $\Delta_{m}=\left[U_{i_{\alpha}} y_{h_{\beta}}\left(\lambda_{m}\right)\right]$ is such that $\left|\operatorname{det} \Delta_{m}\right|$ $\leqq\left|\operatorname{det} \delta_{m}\right|$ and, moreover, lim $\operatorname{det} \Delta_{m} / \operatorname{det} \delta_{m}$ exists, in fact is bounded by one. As a matter of convenience it is assumed that $i_{1}=j_{1}=1, \cdots$, $i_{n-k}=j_{n-k}=n-k$ so that $\delta_{m}=\left[U_{i} y_{j}\left(\lambda_{m}\right)\right], i, j=1, \cdots, n-k$. Now, with $y_{j}=\left(y_{1 j}, \cdots, y_{n j}\right)$, consider the $k$ infinite sequences of vectors $\left\{x_{j}\left(t, \lambda_{m}\right)\right\}, j=1, \cdots, k$, where for each $j$ the $n$ components $x_{1 j}$, $\cdots, x_{n j}$ of $x_{j}$ are determinants of order $n-k+1$ defined by

$$
x_{i j}\left(t, \lambda_{m}\right)=\left|\begin{array}{cccc}
y_{i 1}\left(t, \lambda_{m}\right) & \cdots & y_{i n-k}\left(t, \lambda_{m}\right) & c_{m} y_{i n-k+j}\left(t, \lambda_{m}\right) \\
U_{1} y_{1}\left(\lambda_{m}\right) & \cdots & U_{1} y_{n-k}\left(\lambda_{m}\right) & c_{m} U_{1} y_{n-k+j}\left(\lambda_{m}\right) \\
\dot{U}_{n-k} y_{1}\left(\lambda_{m}\right) & \cdots & U_{n-k} y_{n-k}\left(\lambda_{m}\right) & c_{m} U_{n-k} y_{n-k+j}\left(\lambda_{m}\right)
\end{array}\right|
$$

with $c_{m}=(-1)^{n-k} / \operatorname{det} \delta_{m}$. Clearly, $x_{1}\left(t, \lambda_{m}\right), \cdots, x_{k}\left(t, \lambda_{m}\right)$ are linearly independent solutions of (1), (2) for $\lambda=\lambda_{m}$ inasmuch as each $x_{j}$ is a linear combination of $y_{1}, \cdots, y_{n-k}, y_{n-k+j}$ with the coefficient of $y_{n-k+j}$ equal to one. Moreover, $\lim x_{j}\left(t, \lambda_{m}\right)$ exists; indeed, because

$$
\begin{aligned}
x_{j}(t) & =\lim x_{j}\left(t, \lambda_{m}\right) \\
& =\gamma_{j 1} y_{1}\left(t, \lambda_{0}\right)+\cdots+\gamma_{j n-k} y_{n-k}\left(t, \lambda_{0}\right)+y_{n-k+j}\left(t, \lambda_{0}\right)
\end{aligned}
$$

for some $\gamma_{j 1}, \cdots, \gamma_{j n-k}$, it follows that $x_{1}(t), \cdots, x_{k}(t)$ are linearly independent solutions of (1), (2) for $\lambda=\lambda_{0}$ and that the convergence is uniform on $[a, b]$, completing the proof.

Let $p_{1}, \cdots, p_{n}$ be complex valued functions continuous in $(t, \lambda)$ for $t \in[a, b]$ and $\lambda \in R$. Theorem 2 below concerns itself with the $n$th order linear scalar differential equation 


$$
y^{(n)}+p_{1} y^{(n-1)}+\cdots+p_{n} y=0
$$

subject to (2). Its proof reflects a simple application of Theorem 1.

THEOREM 2. Suppose that $\int_{a}^{b} u(t) \bar{v}(t) d t=0$ for every pair of solutions $u$, $v$ of (3), (2) corresponding to distinct values of $\lambda$. Then there exists a deleted neighborhood of $\lambda_{0}$ at each $\lambda$ of which the compatibility of (3), (2) is zero.

Proof. If the theorem is false, Theorem 1 then guarantees the existence of an infinite sequence $\left\{\lambda_{m}\right\}$ of distinct values of $\lambda$ converging to $\lambda_{0}$ and a sequence $\left\{x_{m}\right\}$ of solutions of (3), (2) for $\lambda=\lambda_{m}$ converging uniformly on $[a, b]$ to $x$, a solution of (3), (2) for $\lambda=\lambda_{0}$ which is not the trivial solution. But $\int_{a}^{b} x \bar{x}_{m}=0$ for every $m$ together with uniform convergence implies $\int_{a}^{b} x \bar{x}=0$, an impossibility.

COROLlary. The eigenvalues of a regular selfadjoint problem on a finite interval are isolated.

\section{REFERENCES}

1. Maxime Bocher, On a small variation which renders a ïnear differential system incompatible, Bull. Amer. Math. Soc. 21 (1914), 1-6.

2. W. M. Whyburn, On the Green's function for systems of differential equations, Ann. of Math. 28 (1927), 291-300.

3. - Differential systems with boundary conditions at more than two points, Proc. Conference Differential Equations, Univ. of Maryland Bookstore, College Park, Md., 1956, pp. 1-21.

UNIVERSITY of Delaware 